$\overline{\text { Original }}$

\title{
Molecular Epidemiological Analysis of Methicillin- Resistant Staphylococci in a Neonatal Intensive Care Unit
}

\author{
YASUSHI TAKEI ${ }^{1,2 *}$, KUMI YOKOYAMA ${ }^{3}$, HIDEKI KATANO $^{4}$, \\ MAMI TSUKIJI ${ }^{5}$, AND TAKAYUKI EZAKI ${ }^{1}$ \\ 'Department of Microbiology, Gifu University, Graduate School of Medicine, \\ 1-1 Yanagido, Gifu-shi, Gifu 501-1193, Japan \\ ${ }^{2}$ Gifu University of Medical Science, 795-1 Nagamine Ichihiraga, Seki-shi, Gifu 501-3892, Japan \\ ${ }^{3}$ Tokyo Healthcare University, 4-1-17 Higashigotannda, Shinagawa, Tokyo 141-8648, Japan \\ ${ }^{4}$ Venex Research Institute, 1-21-8 Asahi bldg 2F Asahicho, Atsugi-shi, Kanagawa 243-0014, Japan \\ ${ }^{5}$ Kanagawa Prefectural University of Human Service, 1-10-1 Heiseimachi, \\ Yokosuka-shi, Kanagawa 238-8522, Japan
}

Received 13 September, 2010/Accepted 27 October, 2010

To investigate the contamination by and transmission of MRSA/MRCNS in the old NICU of Hospital A and the relocated NICU (new NICU), we isolated and evaluated staphylococci from nurses' palms, towels under the heads of neonates, infant incubators (including portholes and infant covers), and room air. Detection rates of MRSA/MRCNS isolated from different sample locations were $52.6 \%$ in the old NICU and $53.4 \%$ in the new NICU, which demonstrates that the nurses' palms in both the old and new NICUs and indoor environment were contaminated with MRSA/MRCNS. In the old NICU, numerous MRSA and MRCNS strains (Log $3.17 \pm 0.19 \mathrm{cfu} / 10$ $\mathrm{cm}^{2}$ ) were identified from towels, and the implementation of improvement plans resulted in a decrease in the number of MRSA/MRCNS isolates (Log $1.95 \pm 0.57 \mathrm{cfu} / 10 \mathrm{~cm}^{2}$ ) from the towels used in the new NICU. A homology study of MRSA/MRCNS strains by PFGE DNA restriction patterns identified genotypes that showed similar patterns in the nurses' palms, towels, infant incubators, and room air.

Key words : NICU/Methicillin-resistant Staphylococcus aureus/Methicillin-resistant coagulasenegative Staphylococci/Pulsed field gel electrophoresis.

\section{INTRODUCTION}

Major organisms that cause nosocomial infection include methicillin-resistant Staphylococcus aureus (MRSA) and methicillin-resistant coagulase-negative Staphylococci (MRCNS), which are resistant to many antimicrobial agents, including $\beta$-lactam antibiotics (Kitamoto et al., 2005; Kobayashi, 1992). Similar to methicillin sensitive Staphylococcus aureus, MRSA can be found on the human skin, and in the nasal cavity and intestinal tract. It causes suppurative inflammation that is manifested as localized abscesses, as

* Corresponding author. Tel:+81-575-22-9401 ext.804 Fax : +81-575-23-0884. E-mail: ytakei(a)u-gifu-ms.ac.jp well as food poisoning, scalded skin syndrome, and toxic shock syndrome by producing toxins, but MRSA has been considered less likely to cause serious infections in healthy hosts with normal immunity (Nasu, 1992). However, MRSA causes severe infection in immune system-compromised hosts such as elderly patients or premature infants (Kitamoto et al., 2005; Matsumura et al., 1999). MRCNS, with lower pathogenicity than MRSA, cause infectious endocarditis, urinary tract infection, and sepsis in compromised hosts via catheters from the blood vessels and urinary tract, and poses senous clinical concerns (Kobayashi, 1992; Fleer et al., 1986).

The route of nosocomial infection includes endogenous infections caused by microorganisms that 
inhabit the patients' pharynx, nasal cavity, and skin, and exogenous infections from other patients and medical personnel or via medical equipment. In particular, exogenous infection, the main cause of nosocomial infection, are considered to be caused by direct contact with the hands of medical personnel (Kitamoto et al., 2005; Komori et al., 2003; Senda, 1998).

Neonatal infants with immature immune systems who are treated and monitored in neonatal intensive care units (NICU) are susceptible to infection with microorganisms (Takemura et al., 2002; Hasegawa et al., 2003). Infectious diseases caused by multiple drug-resistant bacteria such as MRSA/MRCNS are likely to be resistant to many antimicrobial agents (Sunakawa et al., 1992). In the NICU, patients are not in frequent contact with other patients and do not move around, so it is suspected that exogenous infections caused by multiple drug-resistant bacteria such as MRSA/MRCNS are direct infections via medical personnel (Takemura et al., 2002; Hasegawa et al., 2003).

In this study, we isolated and evaluated staphylococci from nurses' palms, towels under the heads of neonates in infant incubators, infant incubators (including portholes and infant covers), and room air using drug susceptibility testing, the polymerase chain reaction ( $P C R$ ) method, and the pulsed field gel electrophoresis (PFGE) method to investigate the contamination by and transmission of MRSA/MRCNS in the old NICU of Hospital A and the relocated NICU (new NICU).

\section{MATERIALS AND METHODS}

\section{Duration of the study}

The study was conducted at the old NICU between July 4, 2005 and August 31, 2005, and at the new NICU between July 3, 2006 and July 16, 2006.

\section{Study subjects}

Samples were taken from both palms of 29 nurses of the old NICU and 41 nurses of the new NICU (including 29 nurses of the old NICU) who agreed to participate in this experiment, from portholes of infant incubators, infant covers to protect neonates from the light in infant incubators, and towels under the heads of neonates in the old and new NICUs, and from the room air in the old and new NICUs.

\section{Sample collection}

Nurses' palms

Samples from nurses' palms were collected by the stamp method, in which the nurses pressed the palms of both hands immediately after nursing care activities onto egg-yolk mannit-NaCl-agar media (Eiken Chemical Co., Ltd.) for the selective isolation of staphylococci.

Towels and infant incubators

Samples from the towels under the heads of neonates were collected using the standard agar stamp media (Nissui Pharmaceutical Co., Ltd.) to estimate the total number of bacteria and TGSE agar stamp media (Nissui Pharmaceutical Co., Ltd.) to selectively isolate staphylococci. Samples from the infant incubators (including portholes and infant covers) were collected by the stamp method using TGSE agar stamp media. It should be noted that the samples of the towels under the heads of neonates were collected after completion of the night shift and before the towels were changed during the day shift.

Room air in the old and new NICUs

Samples of the room air in the old and new NICUs were collected using an air sampler (International $\mathrm{PBI}$, Italy) every day for 2 weeks. The air sampler was placed $120 \mathrm{~cm}$ from the floor and it collected airborne bacteria by allowing the passage of 1,000 liters of air for 10 minutes and using egg-yolk mannit-NaCl-agar media (Nissui Pharmaceutical Co., Ltd.) for the selective isolation of staphylococci. The samples were collected between 16:00 and 17:00 (old NICU), and between 10:00 and 13:00 (new NICU) outside the visiting hours for family members to avoid any influence of visitors from outside the hospital or humanmediated air dispersion.

Isolation and cultivation of bacteria

The media containing the samples were incubated aerobically at $37^{\circ} \mathrm{C}$ for 48 hours, and 1 to 4 macroscopically different colonies were harvested and Gram stained. Then, only gram positive cocci were selected and identified as staphylococci by using an identification kit, API Staph (SYSMEX bioMérieux Co., Ltd.).

\section{Drug susceptibility testing}

The testing was performed by the single disc method using the SN Disc (Nissui Pharmaceutical Co., Ltd.). The drugs used were oxacillin (MPIPC), clindamycin (CLDM), erythromycin (EM), fosfomycin (FOM), gentamicin (GM), minocycline (MINO), and norfloxacin (NFLX). In this experiment, strains resistant to MPIPC were considered as methicillinresistant staphylococci.

\section{Genotyping of methicillin-resistant staphylo- cocci}

Colony-direct (CD)-PCR, in which colonies were added directly to PCR reactions, was used to identify 
the methicillin resistance gene mec A that was detected by drug susceptibility testing (Tsuchizaki et al., 2000). Specifically, methicillin-resistant staphylococci identified by drug susceptibility testing were isolated and cultured on heart infusion agar plates (HI; Nissui Pharmaceutical Co., Ltd.). The tip of a sterile toothpick was applied to grown colonies, and then the harvested bacteria were added directly to PCR reactions. A PCR thermal cycler (Gene Amp PCR System 2400; PERKIN ELMER, USA) was used. The PCR was performed using 30 cycles with denaturation at $94^{\circ} \mathrm{C}$ for $30 \mathrm{sec}$ and annealing at 55 ${ }^{\circ} \mathrm{C}$ for $30 \mathrm{sec}$, followed by extension at $72^{\circ} \mathrm{C}$ for $1 \mathrm{~min}$. Under Gelstar $^{\circledR}$ (TaKaRa) staining, strains that were found to carry the PCR-amplified resistance gene mec A at $623 \mathrm{bp}$ were considered as methicillinresistant staphylococci (Fig. 1). The PCR primers for the resistance gene mec $A$ used in this study were 5 ' -GGTGGTTACAACGTTACAAG-3 ' and 5' GCATTGTAGCTAGCCATTCC-3' .

Genotype analysis of methicillin-resistant staphylococci in which the resistance gene mec A was identified by the CD-PCR was performed using the methods of Mitsuda et al. (1995) and Tomoda et al. (1996). Specifically, methicillin-resistant staphylococci were incubated at $37^{\circ} \mathrm{C}$ for $24 \mathrm{~h}$ on $\mathrm{HI}$ liquid media (Nissui Pharmaceutical Co., Ltd.), bacteria were collected by centrifugation, and then a sample block was created. The bacteria embedded in the sample block were lysed with lysozyme (Wako Pure Chemical Industries, Ltd.) and lysostaphin (Wako Pure Chemical Industries, Ltd.) and treated with proteinase K (Wako Pure Chemical Industries, Ltd.). The sample block was washed and chromosomal DNA was cut with the restriction enzyme Smal (TaKaRa), and then the sample block was inserted

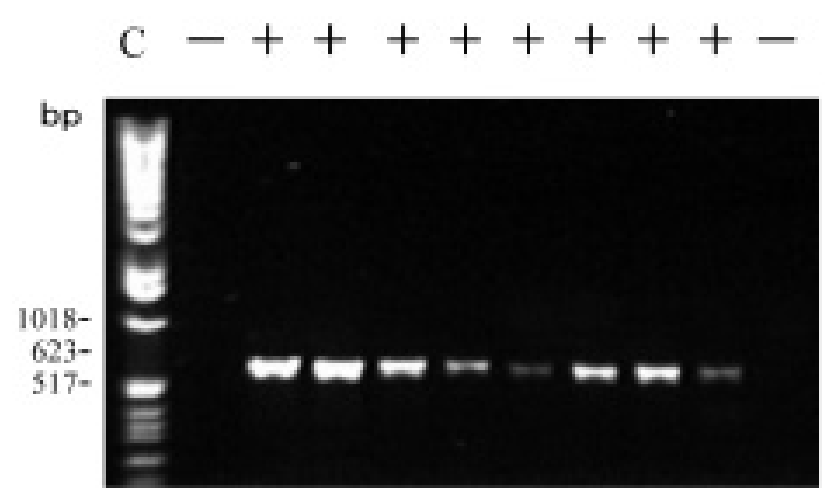

FIG. 1. Detection of the resistance gene mec A using CDPCR. C : 1Kb DNA ladder, bp : base pair, + : mec A positive, - : mec A negative into a sample slot in 1\% agarose for electrophoresis (TaKaRa), and electrophoresis was performed using Genofield (AE-8900 : ATTO). Electrophoresis was carried out at a direct current (DC) of $51 \mathrm{~V}$, alternating current (AC) of $307 \mathrm{~V}, \mathrm{AC}$ frequency of $0.01 \mathrm{~Hz}$ (Start), and $0.2 \mathrm{~Hz}$ (End), with a migration time of 16 hours. Homology between strains was classified (genotyping) according to the size of $48.5-485 \mathrm{~kb}$ and the number of bands (at least 10) by using a $\lambda$ ladder (BIO-RAD) as a molecular-weight marker (Tenover et al., 1995).

\section{Consideration of infection control practices}

Results of the investigation of the MRSA/MRCNS isolated from the samples collected in the old NICU were reported to the nursing staff of the old NICU of this hospital, and problems in conventional infection control practices were pointed out, and researchers discussed improvement plans and activities with the nursing staff of the old NICU.

\section{Statistical analysis}

The number of bacteria on the towels under the heads of neonates used in the old and new NICUs was compared between the two groups using Welch's $t$-test. The Statistical Package for the Social Sciences (SPSS) for Windows11.5J was used for the analysis.

\section{Ethical considerations}

The subjects of this study were informed of the purpose of this study, that participation was voluntary and that they would not suffer any disadvantages even if they did not participate, and that they could refuse to cooperate with the study. They were also told how their privacy would be protected, and their cooperation was requested. Submission of a hand stamp medium was considered as consent to participate.

\section{RESULTS}

\section{Methicillin-resistant staphylococci isolated from the nurses' palms}

A total of 149 strains of staphylococci were isolated from the nurses' palms in the old NICU. Screening assays for drug susceptibility testing and CD-PCR demonstrated 81 strains of methicillin-resistant staphylococci had the resistance gene mec $A$, of which 6 strains were resistant to MRSA and 75 were resistant to MRCNS (Table 1).

The PFGE restriction patterns of MRSA/MRCNS isolated from all samples in the old NICU were classified into 17 groups (Fig. 2). Of these, the restriction patterns of methicillin-resistant staphylococci isolated 
TABLE 1. Drug susceptibility patterns of staphylococci isolated from different sample locations in the old NICU.

\begin{tabular}{|c|c|c|c|c|c|c|c|c|c|c|c|}
\hline \multicolumn{8}{|c|}{ Drug } & \multicolumn{4}{|c|}{ Sample locations } \\
\hline No. & MPIPC & CLDM & EM & MINO & GM & FOM & NFLX & $\begin{array}{c}\text { Nurses' } \\
\text { palms }\end{array}$ & $\begin{array}{c}\text { Towels under } \\
\text { the heads of } \\
\text { neonates }\end{array}$ & $\begin{array}{l}\text { infant incubators } \\
\text { (including portholes } \\
\text { and infant covers) }\end{array}$ & $\begin{array}{l}\text { Room } \\
\text { air }\end{array}$ \\
\hline 1 & - & - & - & + & - & - & - & $45(5)$ & $20(2)$ & 29 & 6 \\
\hline 2 & - & - & - & + & + & - & - & 0 & 0 & $2(2)$ & $3(2)$ \\
\hline 3 & - & - & - & + & + & - & + & 2 & 0 & 1 & 2 \\
\hline 4 & - & - & - & + & + & + & + & 3 & 0 & 0 & 0 \\
\hline 5 & - & + & + & + & - & + & + & 3 & 0 & 0 & 5 \\
\hline 6 & - & + & + & + & + & + & - & 2 & 0 & 0 & 0 \\
\hline 7 & - & + & - & + & - & + & - & 0 & 0 & 0 & 1 \\
\hline 8 & - & + & + & + & + & + & + & $7(1)$ & 0 & 0 & 1 \\
\hline 9 & - & - & - & + & - & - & + & 0 & 0 & 1 & 0 \\
\hline 10 & - & + & + & + & - & - & - & 0 & 1 & 1 & 0 \\
\hline 11 & - & + & + & + & - & + & - & 6 & 2 & 4 & 0 \\
\hline 12 & - & - & - & + & + & + & - & 0 & 0 & 2 & 0 \\
\hline 13 & - & + & - & + & - & - & - & 0 & 1 & 2 & 0 \\
\hline 14 & - & - & - & + & - & + & + & 0 & 2 & $5(2)$ & 0 \\
\hline 15 & - & + & - & + & + & + & + & 4 & 4 & 4 & 0 \\
\hline 16 & - & - & - & + & - & + & - & 3 & 0 & $1(1)$ & 0 \\
\hline 17 & - & - & - & - & - & - & - & 1 & 0 & 2 & 0 \\
\hline 18 & - & + & + & + & + & - & + & 3 & 0 & 0 & 0 \\
\hline 19 & - & - & + & + & - & - & - & 2 & 0 & 0 & 0 \\
\hline
\end{tabular}

No : Numbers according to drug susceptibility patterns, MPIPC : Oxacillin, CLDM : Clindamycin, EM : Erythromycin, MINO : Minomycin, GM : Gentamicin, FOM : Fosfomycin, NFLX : Norfloxacin, + : Drug susceptibility,

- : Drug resistance, The number is the number of the methicillin-resistant staphylococci strains, ( ) The number is the number of the MRSA strains

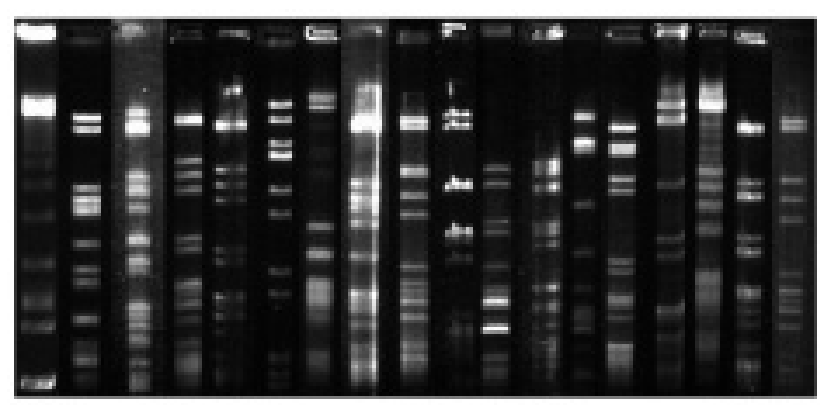

(M) A $A^{*}$ B B B C C D E F G H I J K L M N

FIG. 2. PFGE restriction patterns of MRSA/MRCNS strains isolated from the samples in the old NICU. Restriction enzyme : Sma I, (M): Molecular-weight marker ( $\lambda$ ladder : 48.5 485kb), A N : Genotype

from the nurses' palms in the old NICU were classified into 14 groups (A-F, I-M). Sixty-six point six percent (4/6) of the MRSA showed Pattern C, and $44 \%$ (33/75) and 44\% (33/75) of the MRCNS showed Pattern A" and Pattern B, respectively (Fig. 3, 4).

A total of 298 strains of staphylococci were isolated from the nurses' palms in the new NICU. Screening assays for drug susceptibility testing and CD-PCR demonstrated 159 strains of methicillin-resistant

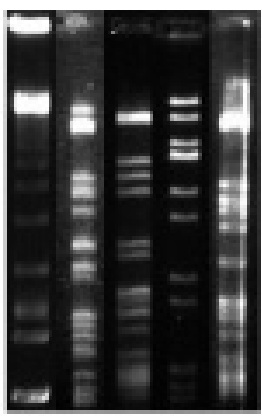

(Mi) $\mathrm{A} * \mathrm{~B}$ C D

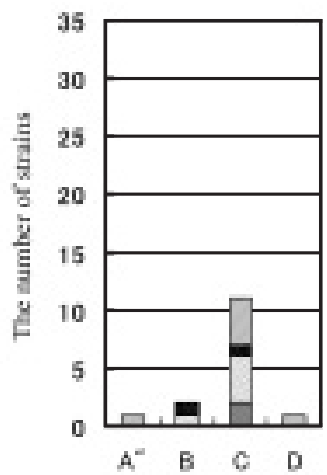

FIG. 3. PFGE restriction patterns and the number of MRSA strains isolated from the samples in the old NICU. $\square$ : Nurses' palms, $\square$ : Towels under the heads of neonates, $\square$ : Infant incubators (including portholes and infant covers), $\square$ : Room air, (M): Molecular-weight marker ( $\lambda$ ladder: 48.5 485kb), A", B, C, D : Genotype

staphylococci had the resistance gene mec $A$, of which 21 strains were resistant to MRSA and 138 were resistant to MRCNS (Table 2).

The PFGE restriction patterns of MRSA/MRCNS isolated from all samples in the new NICU were classified into 14 groups (Fig. 5). Of these, the restriction patterns of methicillin-resistant staphylococci isolated from the nurses' palms in the new NICU were 
TABLE 2. Drug susceptibility patterns of staphylococci isolated from different sample locations in the new NICU.

\begin{tabular}{|c|c|c|c|c|c|c|c|c|c|c|c|}
\hline \multicolumn{8}{|c|}{ Drug } & \multicolumn{4}{|c|}{ Sample locations } \\
\hline No. & MPIPC & CLDM & EM & MINO & GM & FOM & NFLX & $\begin{array}{r}\text { Nurses' } \\
\text { palms }\end{array}$ & $\begin{array}{c}\text { Towels under } \\
\text { the heads of } \\
\text { neonates }\end{array}$ & $\begin{array}{l}\text { infant incubators } \\
\text { (including portholes } \\
\text { and infant covers) }\end{array}$ & $\begin{array}{c}\text { Room } \\
\text { air }\end{array}$ \\
\hline 1 & - & - & - & + & - & - & - & $25(2)$ & 7 & 3 & $6(1)$ \\
\hline 2 & - & - & - & + & + & - & - & $9(7)$ & 0 & 0 & 0 \\
\hline 3 & - & - & - & + & + & - & + & 3 & 0 & 0 & 0 \\
\hline 4 & - & - & - & + & + & + & + & 1 & 0 & 0 & 0 \\
\hline 5 & - & + & + & + & - & + & + & 1 & 0 & 0 & 1 \\
\hline 6 & - & + & + & + & + & + & - & 1 & 0 & 0 & 0 \\
\hline 7 & - & + & - & + & - & + & - & 7 & 0 & 0 & 0 \\
\hline 8 & - & + & + & + & + & + & + & 10 & $1(1)$ & 2 & 0 \\
\hline 9 & - & - & - & + & - & - & + & 28 & 1 & 0 & $2(1)$ \\
\hline 10 & - & + & + & + & - & - & - & 9 & 1 & 2 & 0 \\
\hline 11 & - & + & + & + & - & + & - & 1 & 0 & 0 & 0 \\
\hline 12 & - & - & - & + & + & + & - & 2 & 0 & 1 & 0 \\
\hline 13 & - & + & - & + & - & - & - & 10 & 10 & 0 & 2 \\
\hline 14 & - & - & - & + & - & + & + & 10 & 1 & 1 & 1 \\
\hline 15 & - & + & - & + & + & + & + & 7 & 0 & 3 & 2 \\
\hline 16 & - & - & - & + & - & + & - & $6(1)$ & 0 & $2(1)$ & 0 \\
\hline 17 & - & - & - & - & - & - & - & $1(1)$ & 0 & 0 & 0 \\
\hline 18 & - & + & - & + & + & + & - & 10 & 0 & 0 & 2 \\
\hline 19 & - & - & + & + & - & - & - & 0 & 0 & 1 & 0 \\
\hline 20 & - & + & - & + & - & - & + & 1 & 0 & 1 & 0 \\
\hline 21 & - & + & - & + & - & + & + & 5 & 0 & 0 & 0 \\
\hline 22 & - & - & - & - & + & - & - & $12(10)$ & 0 & 0 & 0 \\
\hline
\end{tabular}

No : Numbers according to drug susceptibility patterns, MPIPC : Oxacillin, CLDM : Clindamycin, EM : Erythromycin, MINO : Minomycin, GM : Gentamicin, FOM : Fosfomycin, NFLX : Norfloxacin, + : Drug susceptibility,

- : Drug resistance, The number is the number of the methicillin-resistant staphylococci strains, $(\quad)$ The number is the number of the MRSA strains

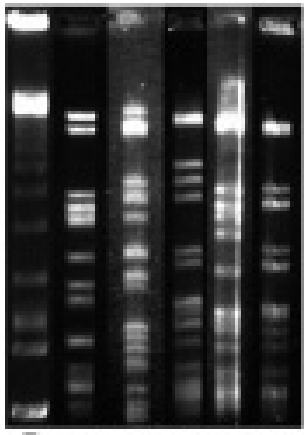

(M) A A"B D M

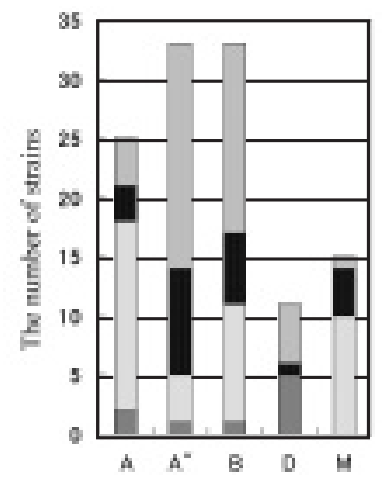

FIG. 4. PFGE restriction patterns and the number of major MRCNS strains isolated from the samples in the old NICU. $\square$ : Nurses' palms, $\square$ : Towels under the heads of neonates, $\square$ : Infant incubators (including portholes and infant covers), $\square$ : Room air, (M): Molecular-weight marker ( $\lambda$ ladder: 48.5 485kb), A, A", B, D, M : Geno type

classified into 12 groups (A-C", E, I, J, N, O, and Q). Sixty-six point six percent (4/6) of the MRSA showed Pattern C, and 25\% (14/56) and 17.8\% (10/56) of the MRCNS showed Pattern B and Pattern C, respectively (Fig. 6, 7). In 97 strains, fewer than 10 bands

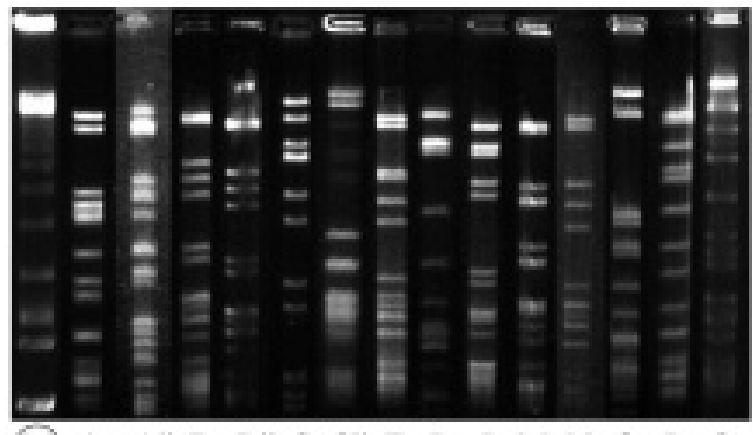

(M) $\mathrm{A} \mathrm{A}^{*} \mathrm{~B} \mathrm{~B}^{*} \mathrm{C} \mathrm{C}^{*} \mathrm{E} / \mathrm{J} \mathrm{M}$ N O $\mathrm{P} \quad \mathrm{Q}$

FIG. 5. PFGE restriction patterns of MRSA/MRCNS strains isolated from the samples in the new NICU. Restriction enzyme : Sma | (M): Molecular-weight marker ( $\lambda$ ladder: 48.5 485kb) A C", E, I, J, M Q : Genotype

were observed.

Methicillin-resistant staphylococci isolated from the towels under the heads of neonates in the old and new NICUs

A total of 31 strains of staphylococci were isolated from towels under the heads of neonates in the old 


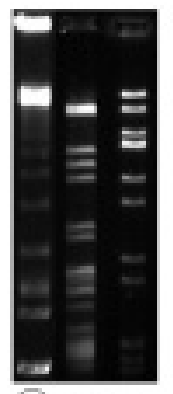

(M) B C

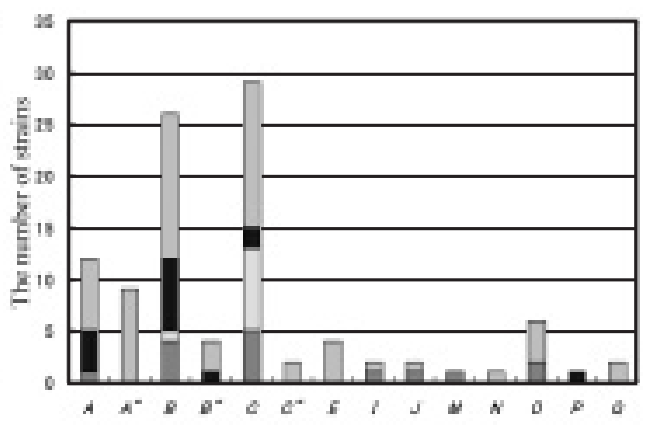

FIG. 6. PFGE restriction patterns and the number of MRSA strains isolated from the samples in the new NICU.

Nurses' palms, : Towels under the heads of neonates, $\square$ : Infant incubators (including portholes and infant covers), $\square$ : Room air, (M): Molecular-weight marker ( $\lambda$ ladder : 48.5 485kb), $A \sim Q$ : Genotype
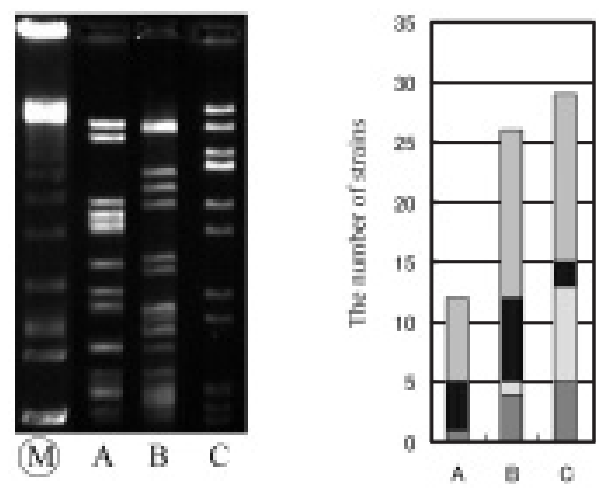

FIG. 7. PFGE restriction patterns and the number of major MRCNS strains isolated from the samples in the new NICU.

: Nurses' palms, $\square$ : Towels under the heads of neonates, $\square$ : Infant incubators (including portholes and infant covers), $\square$ : Room air, (M): Molecular-weight marker ( $\lambda$ ladder : 48.5 485kb), A, B, C : Genotype

NICU. Screening assays for drug susceptibility testing and CD-PCR demonstrated 30 strains of methicillinresistant staphylococci had the resistance gene mec A, of which 2 strains were resistant to MRSA and 28 were resistant to MRCNS (Table 1).

The PFGE restriction patterns of MRSA/MRCNS isolated from the towels under the heads of neonates in the old NICU were classified into 17 groups (Fig. 2). Of these, the restriction patterns of methicillinresistant staphylococci isolated from the towels under the heads of neonates in the old NICU were classified into 8 groups ( $A-B, C-D, M$, and $N$ ). Fifty percent (1/2) of the MRSA showed Pattern $B$ and 50\% Pattern C. 32.1\% (9/28) and 21.4\% (6/28) of MRCNS showed Pattern A" and Pattern B, respectively (Figs. 3 and 4 ).

A total of 30 strains of staphylococci were isolated from the towels under the heads of neonates in the new NICU. Screening assays for drug susceptibility testing and CD-PCR demonstrated 21 strains of methicillin-resistant staphylococci had the resistance gene mec A, of which 1 strain was resistant to MRSA and 20 were resistant to MRCNS (Table 2).

The PFGE restriction patterns of MRSA/MRCNS isolated from the towels under the heads of neonates in the new NICU were classified into 14 groups (Fig. 5). Of these, the restriction patterns of methicillinresistant staphylococci isolated from the towels under the heads of neonates in the new NICU were classified into 5 groups (A, B, B", C, and P). One hundred percent $(1 / 1)$ of the MRSA showed Pattern $C$, and $28.5 \%(4 / 14)$ and $50 \%(7 / 14)$ of the MRCNS showed Pattern A and Pattern B, respectively (Figs. 6 and 7 ). In 6 strains, fewer than 10 bands were observed.

\section{Methicillin-resistant staphylococci isolated from the infant incubators in the old and new NICUs}

A total of 61 strains of staphylococci were isolated from the infant incubators (including portholes and infant covers) in the old NICU. Screening assays for drug susceptibility testing and CD-PCR demonstrated 54 strains of methicillin-resistant staphylococci had the resistance gene mec $\mathrm{A}$, of which 5 strains were resistant to MRSA and 49 were resistant to MRCNS (Table 1).

The FPGE restriction patterns of MRSA/MRCNS isolated from all samples in the old NICU were classified into 17 groups (Fig. 2). Of these, the restriction patterns of methicillin-resistant staphylococci isolated from the infant incubators (including portholes and infant covers) in the old NICU were classified into 8 groups (A-B, C, C", H, M, and N). Eighty percent $(4 / 5)$ of the MRSA showed Pattern B, and $32.6 \%$ (16/49), 20.4\% (10/49) and 20.4\% (10/49) of the MRCNS showed Pattern A, Pattern B, and Pattern M, respectively (Figs. 3 and 4 ).

A total of 34 strains of staphylococci were isolated from the infant incubators (including portholes and infant covers) in the new NICU. Screening assays for drug susceptibility testing and CD-PCR demonstrated 16 strains of methicillin-resistant staphylococci had the resistance gene mec $A$, of which 1 strain was resistant to MRSA and 15 were resistant to MRCNS (Table 2).

The PFGE restriction patterns of MRSA/MRCNS isolated from all samples in the new NICU were classified into 14 groups (Fig. 5). Of these, the restriction patterns of methicillin-resistant staphylococci isolated from infant incubators (including portholes and infant covers) in the new NICU were classified into 2 patterns $(B, C)$. One hundred percent $(1 / 1)$ of the MRSA 
showed Pattern C, and $87.5 \%(7 / 8)$ of the MRCNS showed Pattern C (Figs. 6 and 7). In 7 strains, fewer than 10 bands were observed.

\section{Methicillin-resistant staphylococci isolated from the room air in the old and new NICUs}

A total of 107 strains of staphylococci were isolated from the room air in the old NICU. Screening assays for drug susceptibility testing and CD-PCR demonstrated 18 strains of methicillin-resistant staphylococci had the resistance gene mec $A$, of which 2 strains were resistant to MRSA and 16 were resistant to MRCNS (Table 1).

The PFGE restriction patterns of MRSA/MRCNS isolated from all samples in the old NICU were classified into 17 groups (Fig. 2). Of these, the restriction patterns of methicillin-resistant staphylococci isolated from the room air in the old NICU were classified into 8 groups (A-C, D, G, and H). One hundred percent $(2 / 2)$ of the MRSA showed Pattern C, and $31.2 \%$ $(5 / 16)$ and $31.2 \%(5 / 16)$ showed Pattern $D$ and Pattern $\mathrm{H}$, respectively (Figs. 3 and 4 ).

A total of 54 strains of staphylococci were isolated from the room air in the new NICU. Screening assays for drug susceptibility testing and CD-PCR demonstrated 16 strains of methicillin-resistant staphylococci had the resistance gene mec $A$, of which 2 strains were resistant to MRSA and 14 were resistant to MRCNS.

The PFGE restriction patterns of MRSA/MRCNS isolated from all samples in the new NICU were classified into 14 groups (Fig. 5). Of these, the restriction patterns of methicillin-resistant staphylococci isolated from the room air in the new NICU were classified into 7 groups (A-C, I, J, M, and O). One hundred percent (2/2) of MRSA showed Pattern C, and 30.7\% (4/13) and $23 \%(3 / 13)$ of MRCNS showed Pattern B and Pattern C, respectively (Figs. 6 and 7). In one strain, fewer than 10 bands were observed.

\section{Total number of bacteria detected in the towels under the heads of neonates in the old and new NICUs}

Figure 8 shows the total number of staphylococci detected in the towels under the heads of neonates in the old and new NICUs. The total number of bacteria from the towels under the heads of neonates in the new NICU (Log $1.92 \pm 0.57$ colony forming unit (cfu) $/ 10 \mathrm{~cm}^{2}$ ) was smaller than that in the old $\mathrm{NICU}$ (Log $\left.3.17 \pm 0.19 \mathrm{cfu} / 10 \mathrm{~cm}^{2}\right)(P<0.001)$. The total number of bacteria on the nurses' palms, infant incubators (including portholes and infant covers), and the room air could not be estimated because we used media that was selective for staphylococci alone.

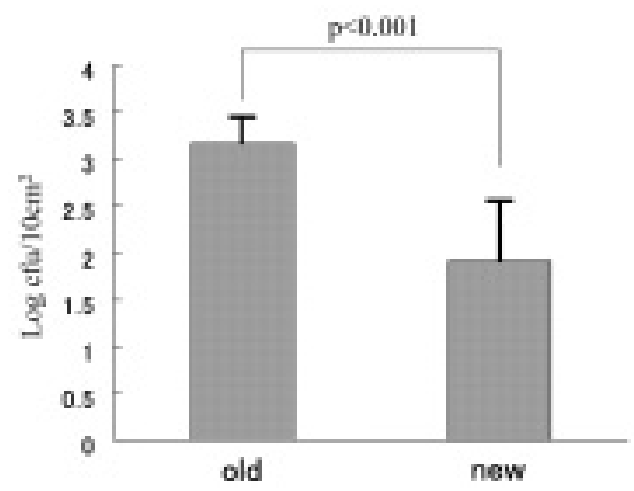

FIG. 8. Total number of bacteria detected in towels under the heads of neonates in the old and new NICUs. Mean, T: Standard deviation, cfu : colony forming unit

\section{Drug susceptibility of MRSA/MRCNS isolated from the nurses' palms, towels, infant incuba- tors, and room air in old and new NICUs}

In the old NICU, 183 strains of MRSA/MRCNS were isolated from the nurses' palms, towels under the heads of neonates, infant incubators (including portholes and infant covers), and room air. Of these, 100 strains (54.6\%) showed resistance only to MINO, which accounted for more than half of the total number of the isolated strains (Table 1).

In the new NICU, 212 strains of MRSA/MRCNS were isolated from the nurses' palms, towels under the heads of neonates, infant incubators (including portholes and infant covers), and room air. Of these, 41 strains (19.3\%) showed resistance only to MINO, which is a decrease compared with the old NICU. However, 12 strains (5.7\%) of bacteria sensitive only to GM were additionally detected, which were not isolated in the old NICU (Table 2).

\section{Correlation between drug susceptibility patterns and PFGE DNA restriction patterns}

In the old and new NICUs, 27, 3, 6, and 4 strains of MRSA were isolated from nurses' palms, towels under the heads of neonates, infant incubators (including portholes and infant covers), and the room air, respectively, and these were identified as separate strains according to the drug susceptibility patterns (Tables 1 and 2). Among these strains of MRSA, 8 strains isolated from the nurses' palms, 2 strains from the towels under the heads of neonates, 5 strains from the infant incubators (including portholes and infant covers), and 4 strains from the room air were identified as identical strains according to the PFGE restriction patterns (Figs. 3 and 6 ). The MRCNS that were identified as strains with different drug susceptibility patterns in the old and new NICUs (Table 1, No. 2-19; Table 2, No. 2-13, and 15-22) included 140 
strains isolated from the nurses' palms, 22 strains from the towels under the heads of neonates, 31 strains from the infant incubators (including portholes and infant covers), and 18 strains from the room air. Among these strains of MRCNS, however, 38 strains isolated from the nurses' palms, 10 strains from the towels under the heads of neonates, 11 strains from the infant incubators (including portholes and infant covers), and 4 strains from the room air were identified as identical strains according to the PFGE restriction patterns. The MRCNS that were identified as identical strains according to the drug susceptibility patterns (Table 1, No. 1; Table 2, Nos. 1 and 14) included 73 strains from the nurses' palms, 26 strains from the towels under the heads of neonates, 33 strains from the infant incubators (including portholes and infant covers), and 12 strains from the room air. However, a homology study of these MRCNS strains by PFGE restriction patterns showed that 27 strains from the nurses' palms, 15 strains from the towels under the heads of neonates, 29 strains from the infant incubators (including portholes and infant covers), and 7 strains from the room air were identified as identical strains.

\section{Homology in restriction patterns of MRSA/MRCNS isolated from the samples col- lected in the old and new NICUs}

The strains of MRSA/MRCNS isolated from the samples collected in the old and new NICUs had 11 common restriction patterns (A, A", B, B", C, C", E, I, $J, M$, and $N$ ), and those in the old NICU had 6 different restriction patterns ( $D, F, G, H, K$, and $L)$ and those in the new NICU had 3 different patterns (O, P, and Q) (Figs. 2 and 5).

\section{Infection control practices}

Results of the examination of the towels under the heads of neonates and the environment in the old NICU were reported to the nursing staff of the old NICU of this hospital, and researchers discussed problems in conventional infection control practices with the nursing staff of the old NICU, who were instructed to implement the following improvement plans and activities:

(1) Changing towels under the heads of neonates once a day

(2) Thorough hand washing and hand disinfection (habitual hand washing, hand disinfection using ethanolbased antiseptic hand rubs before and after care activities, use of gloves during nursing)

(3) To minimize the sharing of scissors, pens, or stethoscopes among nurses.

\section{DISCUSSION}

Prevention of nosocomial infection with MRSA/MRCNS requires accurate knowledge of the sources and routes of infection. In particular, in order to identify MRSA, biological phenotypes have been used based on drug susceptibility patterns, coagulase types, enterotoxin types, and production of toxic shock syndrome toxin. However, the strains of MRSA that were considered to be different strains based on the drug susceptibility patterns, one of the phenotypes examined in this study, were found to be identical strains according to the PFGE analysis. Based on these results and reports of Tomoda et al. (1996) and Sawai et al. (1998), biological phenotypes such as drug susceptibility patterns will produce biased results, and a detailed analysis of sources and routes of infection is extremely difficult. Therefore, molecular biological techniques utilizing genetic characteristics of chromosomal DNA are effective for accurately assessing the true state of nosocomial infection (Komori et al., 2003; Ichiyama and Ohta, 1992; Kobayashi et al., 1997).

In this study, we isolated and evaluated staphylococci from the nurses' palms, towels under the heads of neonates in infant incubators, infant incubators (including portholes and infant covers), and room air using molecular biological techniques of the CD-PCR and the PFGE to investigate the contamination by and transmission of MRSA/MRCNS in the old NICU of Hospital A and the relocated NICU (new NICU).

As shown in Tables 1 (old NICU) and 2 (new $\mathrm{NICU}$ ), numerous MRSA/MRCNS strains were isolated from the nurses' palms, towels under the heads of neonates in infant incubators, infant incubators (including portholes and infant covers), and room air, and it was revealed that the old and new NICUs were contaminated with drug-resistant staphylococci. Contamination with drug-resistant bacteria in hospital rooms is spread by patient-derived drug-resistant bacteria moving upward and downward repeatedly (Ogase et al., 1993). It is suggested that the contamination with MRSA/MRCNS in the old and new NICUs was spread by MRSA and MRCNS derived from nurses' palms, because infants were kept in infant incubators and MRSA/MRCNS strains that showed several DNA restriction patterns were isolated from the nurses' palms in the old and new NICUs (Figs. 3, 4, 6, and 7). We therefore considered it important to educate medical personnel to maintain a basic level of standard precautions and "hand hygiene" for the prevention of nosocomial infection.

The drug susceptibility testing showed that more than half of the MRSA/MRCNS strains isolated from 
the samples collected in the old NICU were resistant only to MINO (Table 1). In the new NICU, the strains resistant only to MINO in the old NICU were decreased, but the strains sensitive only to GM were additionally detected. As a result, it was considered that highly multidrug-resistant MRSA/MRCNS were increased in the new NICU, which could result in a limitation of antimicrobial agents that could be used and in difficulties in treatment.

Based on the results of the PFGE analysis of DNA restriction patterns using genetic characteristics of chromosomal DNA, the MRSA (Figs. 3 and 6) and MRCNS (Figs. 4 and 7) strains that showed an identical restriction pattern were isolated from the nurses' palms, towels under the heads of neonates in infant incubators, infant incubators (including portholes and infant covers), and room air in the old and new NICUs and were considered to be epidemiologically highly related to each other. It was also possible that drugresistant staphylococci were transmitted from the old $\mathrm{NICU}$ to the new NICU via objects or medical personnel. In addition to the common restriction patterns (Figs. 2 and 5), the MRSA/MRCNS strains isolated from the samples in both the old and new NICUs had different restriction patterns. From this it can be inferred that there was a genetic polymorphism of the same strains of staphylococci (Nagase et al., 2002) or the emergence of strains of different origins associated with the relocation of wards or recruitment of new nurses (Kinouchi et al., 1998).

The route of nosocomial infection may be from medical personnel to patients, from patients to medical personnel, or from patients to patients. Indeed, some researchers have reported that medical personnel were associated with cross infection between patients (Senda, 1998; Kiyosuke et al., 2005; Moriwaki, 2003). In our study, numerous strains of MRSA/MRCNS were isolated from the towels under the heads of neonates in infant incubators (Tables 1 and 2), and these strains showed the same restriction pattern as seen in the MRSA (Figs. 3 and 6) and MRCNS (Figs. 4 and 7) strains isolated from the nurses' palms. These results suggest that the MRSA/MRCNS on the towels under the heads of neonates in infant incubators were transmitted via the nurses' palms, because NICU patients are not in frequent contact with other patients and do not move around. Presentation of these results and the improvement plans (e.g. changing towels under the heads of neonates once a day and thorough hand disinfection) to the nursing staff in the old NICU resulted in a decrease in the number of bacteria on the towels under the heads of neonates used in the new NICU. Therefore, to enable nursing staff to carry out effective nosocomial infection control practices, it is important to present real investigation results and discuss improvement plans in response to the results (Kaneko et al., 1992).

It has also been reported that in NICUs, MRSA/MRCNS infection may be transmitted to other patients via the hands of medical personnel who are involved in the treatment and care of the patients carrying resistant bacteria (Komori et al., 2003; Takemura et al., 2002). We did not investigate MRSA/MRCNS from physicians and neonates in this study. A further clarification of this point and a detailed analysis of sources and routes of infection are warranted in the future.

\section{ACKNOWLEDGEMENT}

I am grateful to the director of the NICU, the head of the NICU nurses, and the nursing staff of Hospital A for their assistance in the research associated with this study.

\section{REFERENCES}

Fleer A., Verhoef J., and Hernandez A.P. (1986) Coagulase-negative staphylococci as nosocomial pathogens in neonates. The role of host defense, artificial device, and bacterial hydrophobicity. Am. J. Med., 80, 161165.

Hasegawa Y., Hayano K., Toyoda M., Harada S., Tujii H., Ikuta H., Fujimoto Y., Takeshita S., Oka T., and Ohno S. (2003) Infection Control of MRSA at NICU and Intermediate Care Nursery. (in Japanese), kankyo kansen Zasshi, 18, 333-336.

Ichiyama S., and Ohta M. (1992) Pulsed-field gel electrophoresis for molecular epidemiology of nosocomial infection. (in Japanese) Nippon Rinsho, 50, 143-148.

Kaneko A., Yamazaki T., Tomita F., Baba M., Kojima M., Kawashima C., and Kobayashi I. (1992) Spread of Methicillin-resistant Staphylococcus aureus within the hospital and preventive measures of nosocomial infection. (in Japanese), Kankyo kansen, 7, 15-20.

Kobayashi H. (1992) Coagulase-negative staphylococcal infection. (in Japanese), Nippon Rinsho., 50, 2765-2772.

Kinouchi Y., Suzuki H., and Yatsuyanagi J. (1997) Analysis by PFGE of Legionella pneumophila SG1 divided in Akita. (in Japanese) Annual Report of the Akita Prefectural Institute of Public Health, 41, 27-30.

Kitamoto N., Kato Y., Kanzya S., Katai A., and Tanaka T. (2005) Molecular epidemiology of mechicillin-resistant Staphylococcus aureus (MRSA) by pulsed-field gel electrophoresis. (in Japanese) Kansenshogaku Zasshi, 79, 129-137.

Kiyosuke M., Sato K., Seki M., Takayanagi M., Nagasawa Z., Koguchi A., and Nagayama A. (2005) The Analysis of MRSA strains by PFGE and the infection control strategies in NICU. (in Japanese) Nippon Rinsho Bisebutsugaku Zasshi, 15, 25-31.

Kobayashi M., Ohara-Nemoto Y., and Kaneko M. (1997) Genotyping of clinical isolates of MRSA by PFGE and AP- 
PCR. (in Japanese) Kansenshogaku Zasshi, 71, 620627.

Komori Y., Fukuda N., Ito Y., Kuroki A., and Nikai T. (2003) Sampling of Drug- and disinfectant-resistant microorganisms in the hospital environment, and its effect on the attitude of medical staff. (in Japanese) Kankyo kansen Zasshi, 18, 240-246.

Matsumura K., Takahashi A., and Sakata H. (1999) Colonization of methicillin-resistant Staphylococcus aureus in low-birth-weight infants in a neonatal intensive care unit. (in Japanese) Kankyo kansen, 14, 189-191.

Mitsuda T., Arai k., Kawamoto S., and Yokota S. (1995) Analysis of the molecular epidemiology of the infectious disease by the pulse field gel electrophoresis. (in Japanese) Nippon Saikingaku Zasshi, 50, 1077-1086.

Moriwaki T. (2003) Genotyping of methicillin-resistant Staphylococcus aureus isolated from inpatients and medical workers in orthopaedics ward. (in Japanese) Kansenshogaku Zasshi, 77, 1058-1066.

Nagase N., Sasaki A., Yamashita K., Shimizu A., Wakita Y., Kitai S., and Kawano J. (2002) Isolation and species distribution of staphylococci from animal and human skin. J. Vet. Med. Sci., 64, 245-250.

Nasu Masaru. (1992) Of the anti-MRSA measure, actuallyAn anti-in-hospital infection measure of the MRSA. (in Japanese) Nippon Naika Gakkai Zasshi, 81, 1657-1661.

Ogase H., Matsuura S., Ando S., Yokoyaka H., Teyama S., Inoue K, and Furuhashi M. (1993) The present conditions of the in-hospital infection that I watched from levitation bacteria including the MRSA. (in Japanese) Kankyo Kansen, 8, 85

Sawai T., WANG J., Tomono K., Yanagihara K., Hirakata Y.,
Matsuda J., Mochida C., Tashiro T., Kamihira K., and Kohno S. (1998) Analysis of utility of the phenotyping method on detection of cases infected by multiple strains of methicillin-resistant Staphylococcus aureus (MRSA). (in Japanese) Kansenshogaku Zasshi, 72, 1035-1040.

Senda Y. (1998) Bacteriological and molecular epidemiological investigation on nosocomial infection caused by methicillin-resistant Staphylococcus aureus and its prevention. (in Japanese) Okayama Igakkai Zasshi, 111, 1126.

Sunakawa K., Yokota T., and Nitta Y. (1992) MRSA infections in neonates and premature infants. (in Japanese) Nippon Naika Gakkai Zasshi, 81, 1646-51.

Takemura H., Doi M., Kayano Y., Kawarazaki J., Sugiyama I., Iwamoto Y., Usukura Y., and Shimura K.(2002) Epidemiological study of methicillin-resistant Staphylococcus Strains from NICU. (in Japanese) Kankyo Kansen, 17, 257-263.

Tenover F.C., Arbeit R.D., Goering R.V., Mickelsen P. A., Murray B.E., Persing D.H., and Swaminathan B. (1995) Interpreting chromosomal DNA restriction produced by pulsed-field gel electrophoresis: Criteria for bacterial strain typing. J. Clin. Microbiol., 33, 2233-2239.

Tomoda T., Maeda H., Kitashiba K., Watanabe R., Nomura M., and Yamada Y.(1996) Evaluation of MRSA genomic DNA-RFLP analysis by pulsed-field gel electrophresis for the investigation of infectious route. (in Japanese) Osaka Rosai Hospital Igakuzassi, 20, 57-62.

Tsuchizaki N., Ishikawa J., and Hotta K. (2000) Colony PCR for rapid detection of antibiotic resistance genes in MRSA and enterococci. Jpn. J. Antibiot., 53, 422-429. 